also sparked hope among researchers by showing that a virus injected directly into one tumour could rein in tumours elsewhere in the body.

\section{DEATH BY ASSOCIATION}

The viruses work by generating an immune response. After the virus infects and kills cancer cells, the immune system eliminates the virus and ends up also getting rid of the dead cancer cells. "The side effect from eliminating the virus is that the systemic immune system recognizes the cancer cells," says Tomoki Todo, a neurosurgeon at the University of Tokyo. "Then it starts to attack even those cancer cells that are not infected by the virus."
Scientists reasoned that bolstering such an immune response - for example, by using a checkpoint inhibitor - could amplify this indirect effect. These inhibitors sometimes send cancer into remission for years, but only for a fraction of people.

Studies in mice suggest that combining checkpoint inhibitors with cancer-killing viruses might boost that percentage. And in a small clinical trial involving 21 people with advanced melanoma, Imlygic, together with a checkpoint inhibitor, significantly shrank tumours in $62 \%$ of participants and wiped them out altogether in 33\% (A. Ribas et al. Cell 170, 1109-1119; 2017).
When researchers have combined checkpoint inhibitors with other treatments, they have met with mixed success. Some fervently anticipated combinations have failed in clinical trials. "Could the same thing happen with oncolytic viruses? Sure," says Dmitriy Zamarin, an oncologist at the Memorial Sloan Kettering Cancer Center in New York City.

But Zamarin and others are cautiously optimistic. Many checkpoint-inhibitor combinations target a specific protein, Zamarin notes, whereas oncolytic viruses provoke a much broader immune response that can target cancer in a number of different ways. "That gives us some comfort," he says. -

\title{
Universe's coolest lab set to open up quantum world
}

\author{
NASA's Cold Atom Laboratory will allow physicists to play with quantum phenomena \\ like never before.
}

\section{BY ELIZABETH GIBNEY}

$\longrightarrow$ uantum physicists are about to get their own playground in space. NASA's Cold Atom Laboratory, scheduled to launch to the International Space Station on $20 \mathrm{May}$, is set to be the coldest place in the known Universe. Researchers will use the lab to probe quantum phenomena that would be impossible to observe on Earth.

The US\$83-million mission will be used to study quantum mechanics on the macroscopic scale by making a state of matter known as a Bose-Einstein condensate (BEC). These are clouds of hundreds of thousands of atoms that, when chilled to just above absolute zero, behave as waves that synchronize into a single quantum object. "Just being able to do these experiments in space I think is a huge accomplishment," says Kamal Oudrhiri, mission manager at the Jet Propulsion Laboratory (JPL) in Pasadena, California.

On Earth, gravitational forces usually disperse these condensates within a few seconds. The closest that BECs have come to being in space-like conditions is during brief stints in a research rocket, or falling from a drop tower over 9 seconds. But, floating on the space station, they should be able to exist for at least 10 seconds. That's long enough for them to be cooled to record-low temperatures perhaps as little as 20 trillionths of a degree above absolute zero. That would be the coldest known temperature in the Universe, says
Oudrhiri. Colder and long-lasting condensates will "push the frontiers of studying fundamental physics", says Gretchen Campbell, an atomic physicist at the US National Institute of Standards and Technology in Gaithersburg, Maryland. "It's something people have hoped for for almost 15 years.”

\section{DOWN-SIZED KIT}

Real estate on the space station is at a premium, so engineers had to crunch down atomic-physics equipment that usually fills a large room into a cool-box-sized chest. The equipment will cool rubidium and potassium atoms by scattering laser light off the particles

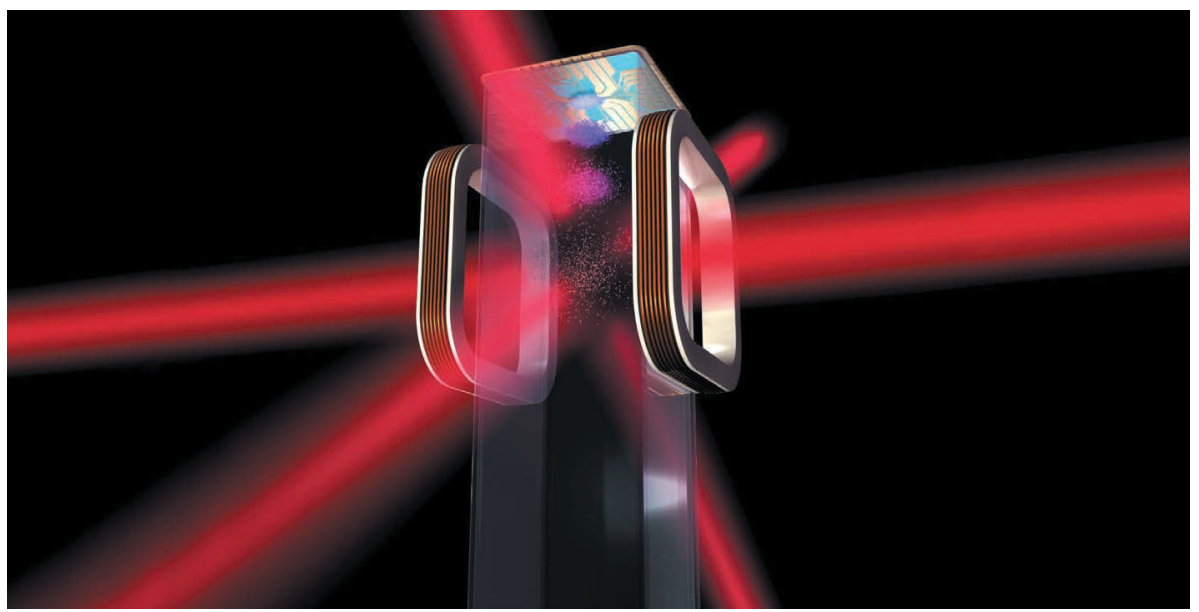

Instruments on NASA's Cold Atom Laboratory will cool atoms to near absolute zero (artist's concept). in all directions to slow them to almost a standstill. It will then use magnetic fields to trap the cloud. To create the condensate, other cooling techniques are used to push the cloud even closer to absolute zero - including creaming off the most energetic atoms using a radio-wave 'knife' and widening the trap to let the cloud expand.

Engineers also had to design shielding to protect the delicate condensates from interference from densely packed components and from Earth's varying magnetic field. Although astronauts will unpack and install the equipment, the experiments will run only while the team is asleep to minimize disruption from 
any movements.

The technology is simpler than initially intended, after a more-complex version of the lab developed a leak that affected the vacuum chamber and threatened to delay the project. So physicists will not yet be able to achieve their ultimate goal of performing spacebased atom interferometry - a process that involves splitting the condensate's quantum wave in two and recombining the waves. The resulting interference patterns would allow scientists to analyse the effects of gravity with exquisite precision, as well as test whether condensates could be used as very sensitive rotation and gravity sensors. But the moreadvanced kit should arrive by the end of 2019, says Robert Thompson, project scientist for the mission at JPL.

\section{BUBBLES, RINGS AND WHIRLPOOLS}

The current iteration will still allow new physics, says Thompson. Five teams are set to experiment with the lab; one plans to use radio waves and magnetic fields to trap the condensate in a bubble shape about 30 micrometres across - roughly half the width of a human hair. Quantum mechanics suggests that because the bubble is both thin and edgeless, the condensate should behave differently from when it is shaped as a disc or sphere on Earth. For instance, it might more

readily form whirlpools known as vortices, says Courtney Lannert, a theoretical physicist at Smith College in Northampton, Massachusetts. On Earth, attempts to create bubbles always end up with bowl shapes as the fluid falls. "We can't get this shape at all unless we can get rid of gravity," she says.

A group led by Eric Cornell of the University of Colorado, Boulder, who won a Nobel prize in 2001 for co-discovering BECs, will try to

"We can't get

this shape at

all unless we

can get rid of gravity." create exotic loosely bound systems known as Efimov states. Named after Russian theoretical physicist Vitaly Efimov, who proposed their existence in 1970, these quantum states crop up where atoms would bind too weakly to join in pairs, but can form threesomes. These are similar to Borromean rings - rings linked in such a way that the system falls apart if any one ring is removed - and are of interest to nuclear physicists because they have parallels with rare and poorly understood three-particle nuclei made up of neutrons and protons. The team hopes to create the simplest Efimov state but also excited, bloated versions in which the atoms bind with each other despite being a bacterium-width apart. The group might also be able to make foursomes of such atoms, known as tetramers, says Maren Mossman, a physicist at Washington State University in Pullman.

Atomic physicists will find the space-station set-up extraordinary for more-practical reasons, says Mossman. They are used to building their own equipment and tweaking experiments as they go. But with the Cold Atom Laboratory, many are sharing a facility for the first time, says Mossman, and they must run experiments through JPL researchers who are operating the facility from the ground. "Folks in particle physics have been doing this since the beginning. But it's so weird to us in atomic physics," she says.

The process worked out "better than most of us expected", says Thompson, who has been working to create such a facility since he joined JPL in 1997. He thinks that the current version is a step towards even more-complex atomic-physics labs in space. NASA is working with the German Aerospace Center (DLR) to build a facility called BECCAL (Bose-Einstein Condensate and Cold Atom Laboratory), he says. Many experiments on the space station already test the effects of low gravity, but for most, the extreme microgravity is "overkill", adds Thompson. "We are one of the experiments that will really highlight what the space station is capable of doing." 